\title{
Low-temperature synthesis and characterization of $\operatorname{Ag}_{2} \mathrm{~S}_{1-x} \mathrm{Te}_{x}(0 \leqslant x \leqslant 1)$
}

\author{
H N VASAN and A K SHUKLA \\ Solid State and Structural Chemistry Unit, Indian Institute of Science, Bangalore 560012 , \\ India \\ MS received 15 January 1992
}

\begin{abstract}
A low-temperature route for the synthesis of $\mathrm{Ag}_{2} \mathrm{~S}, \mathrm{Ag}_{2} \mathrm{Te}$ and their solid solutions $\mathrm{Ag}_{2} \mathrm{~S}_{1-x} \mathrm{Te}_{x}(0 \leqslant x \leqslant 1)$ is reported. $\mathrm{Ag}_{2} \mathrm{~S}$ is prepared by the direct addition of silver nitrate solution to thiourea, while $\mathrm{Ag}_{2} \mathrm{Te}$ is prepared by reacting silver nitrate solution with tellurium in nitric acid and subsequently reducing it with hydrazine hydrate. The solid solutions of $\mathrm{Ag}_{2} \mathrm{~S}$ and $\mathrm{Ag}_{2} \mathrm{Te}$ are obtained by the addition of nitrate solutions of silver and tellurium to thiourea followed by its reduction with hydrazine hydrate. The method enables the synthesis of low-temperature crystalline phase of $\mathrm{Ag}_{2} \mathrm{~S}_{1-x} \mathrm{Te}_{\mathrm{x}}$ solid solutions. The powder $X$-ray diffraction studies suggest that the solid solutions of compositions $x<0.3$ have a phase akin to $\alpha-\mathrm{Ag}_{2} \mathrm{~S}$ and those with compositions $x>0.6$ are similar to $\alpha-\mathrm{Ag}_{2} \mathrm{Te}$. In the intermediate range of compositions $(x=0.4$ and 0.5$)$, the solid solutions are found to be mixtures of $\alpha-\mathrm{Ag}_{2} \mathrm{~S}$ and $\alpha-\mathrm{Ag}_{2} \mathrm{Te}$ phases which transform totally to $\alpha-\mathrm{Ag}_{2} \mathrm{~S}$ phase on prolonged annealing at about $473 \mathrm{~K}$.
\end{abstract}

Keywords. Low-temperature synthesis; silver chalcogenide; $\mathrm{Ag}_{2} \mathrm{X}$ characterization.

\section{Introduction}

Silver chalcogenides, $\mathrm{Ag}_{2} \mathrm{X}(\mathrm{X}=\mathrm{S}$, Se, Te) being mixed conductors are potential materials for certain applications such as solid-state coulometers and battery electrodes. These materials undergo a structural $\alpha$ to $\beta$ phase transition at about $423 \mathrm{~K}$ accompanied by a marked increase in their electronic and ionic conductivities (Wagner 1953; Miyatani 1958, 1959, 1960; Shukla and Schmalzried 1979; Shukla et al 1981; Sohege and Funke 1984, 1989). One could synthesize these chalcogenides by the solid-state reaction of the constituent elements. But a similar method for preparing the solid solutions of $\mathrm{Ag}_{2} \mathrm{~S}$ and $\mathrm{Ag}_{2} \mathrm{Te}$ results either in the formation of an amorphous phase or the high temperature $\beta$-phase which is retained even at room temperature (Miyatani 1960). In this study, we report a low-temperature route to synthesize phases of $\mathrm{Ag}_{2} \mathrm{~S}, \mathrm{Ag}_{2} \mathrm{Te}$ and $\mathrm{Ag}_{2} \mathrm{~S}_{1-x} \mathrm{Te}_{x}(0 \cdot 1 \leqslant x \leqslant 0 \cdot 9)$ similar to the method (Kulifay 1962) for synthesizing transition metal tellurides. These materials have been characterized by elemental analysis, powder X-ray diffraction (PXD) and differential scanning calorimetry (DSC). The room temperature PXD patterns of these solid solutions resemble $\alpha$-phases of either $\mathrm{Ag}_{2} \mathrm{~S}$ or $\mathrm{Ag}_{2} \mathrm{Te}$ depending on their composition. Unlike the parent compounds, which on heating undergo a distinct first order $\alpha-\beta$ transition, the solid solutions show a similar but broad phase transition.

\section{Synthesis and characterization}

Silver sulphide was prepared by mixing the solutions of $\mathrm{AgNO}_{3}\left(2 \times 10^{2} \mathrm{~mol} \mathrm{dm}^{-3}\right)$ and $\mathrm{CS}\left(\mathrm{NH}_{2}\right)_{2}\left(0.1 \mathrm{moldm}^{-3}\right)$ in water and heating around $353 \mathrm{~K}$ with constant 
stirring for 2-3 h. The resulting black precipitate of $\mathrm{Ag}_{2} \mathrm{~S}$ was filtered, washed with water followed by methanol and dried in air oven at about $373 \mathrm{~K}$.

Silver telluride was prepared by dissolving tellurium powder $(0.01 \mathrm{~mol})$ in hot $1: 1$ nitric acid and water. The excess acid was neutralized with the desired quantity of dilute ammonia solution. This solution was then added to silver nitrate $\left(0.2 \mathrm{~mol} \mathrm{dm}^{-3}\right)$ solution. The resulting solution was slowly added with constant stirring to $500 \mathrm{ml}$ of $10 \%$ hydrazine hydrate in water having a pH of 9 which was raised to 10 by adding dilute ammonia solution and heating around $353 \mathrm{~K}$ with constant stirring for about $8 \mathrm{~h}$. The black coloured $\mathrm{Ag}_{2} \mathrm{Te}$ formed was washed and dried as before.

Thiourea hydrolyses in water giving $\mathrm{H}_{2} \mathrm{~S}$ (Pass and Sutcliffe 1968) and $\mathrm{Te}$ in tellurous acid was reduced from +4 to -2 by the addition of hydrazine hydrate. The plausible reaction route for the formation of $\mathrm{Ag}_{2} \mathrm{~S}$ and $\mathrm{Ag}_{2} \mathrm{Te}$ may then be written as,

$$
\begin{aligned}
\mathrm{S}= & \mathrm{C}\left(\mathrm{NH}_{2}\right)_{2}+2 \mathrm{H}_{2} \mathrm{O} \rightarrow 2 \mathrm{NH}_{3}+\mathrm{H}_{2} \mathrm{~S}+\mathrm{CO}_{2} \\
& 2 \mathrm{AgNO}_{3}+\mathrm{H}_{2} \mathrm{~S} \rightarrow \mathrm{Ag}_{2} \mathrm{~S}+2 \mathrm{HNO}_{3} \\
& 2 \mathrm{Ag}^{+}+\mathrm{Te}^{+4}+\mathrm{N}_{2} \mathrm{H}_{4}+\mathrm{OH}^{-} \rightarrow \mathrm{Ag}_{2} \mathrm{Te}+\mathrm{N}_{2} \mathrm{O}+5 \mathrm{H}_{2} \mathrm{O} .
\end{aligned}
$$

A similar procedure was adopted for the preparation of solid solutions $\mathrm{Ag}_{2} \mathrm{~S}_{1-x} \mathrm{Te}_{\boldsymbol{x}}$ $(0 \cdot 1 \leqslant x \leqslant 0.9)$. Solutions of thiourea, tellurous acid and silver nitrate were added in the molar ratio of $1-x: x: 2$ to $500 \mathrm{ml}$ of $10 \%$ hydrazine hydrate solution in water at $\mathrm{pH}$ equal to 10 . A portion of these compounds were annealed around $473 \mathrm{~K}$ in evacuated $\left(10^{-5}\right.$ torr) sealed tubes. Both the unannealed and annealed samples were characterized by PXD and DSC. The room temperature PXD patterns were recorded on a JEOL JDX-8P/70 X-ray diffractometer with $\mathrm{CuK}_{\alpha}(\lambda=1.5418 \AA)$ radiation. While the high temperature PXD patterns were obtained using a locally fabricated high temperature cell attached to PW 1050/70 Phillips X-ray diffractometer. The phase transformation temperature of the solid solutions were recorded on a Perkin Elmer DSC-2 differential scanning calorimeter.

\section{Results and discussion}

The elemental analysis showed a slight excess of $\mathrm{Ag}$ in all the solid solutions and hence only the nominal compositions are reported. The indexed room temperature PXD patterns of unannealed $\mathrm{Ag}_{2} \mathrm{~S}, \mathrm{Ag}_{2} \mathrm{Te}$ and two representative solid solutions $\mathrm{Ag}_{2} \mathrm{~S}_{1-x} \mathrm{Te}_{x}(x=0.2$ and 0.8$)$ are shown in figures 1 and 2 and their indexed patterns with relative intensities are given in tables 1 and 2 , respectively. The PXD patterns of $\mathrm{Ag}_{2} \mathrm{~S}$ and $\mathrm{Ag}_{2} \mathrm{Te}$ match with the respective monoclinic $\alpha$-phases, whereas their solid solutions with compositions $x<0.3$ are similar to $\alpha-\mathrm{Ag}_{2} \mathrm{~S}$ phase and those of compositions $x>0.6$ are akin to $\alpha-\mathrm{Ag}_{2}$ Te phase. In the intermediate range $(x=0.4$, $0.5)$, the products are mixtures of $\alpha-\mathrm{Ag}_{2} \mathrm{~S}$ and $\alpha-\mathrm{Ag}_{2} \mathrm{Te}$ phases.

From tables 3 and 4 , it is seen that within a system the lattice parameters of the solid solutions do not vary much in relation to their parent compounds although the atomic sizes of $\mathrm{S}(1.27 \AA)$ and $\mathrm{Te}(1.60 \AA)$ do differ. However, the solid solutions have different phase transition temperatures as seen from figure 3 . The phase transformation temperatures of the parent compounds are in agreement with the values reported in the literature (Miyatani 1959). The phase transition temperature of the solid solutions continuously varies on the tellurium and sulphur-rich sides. 


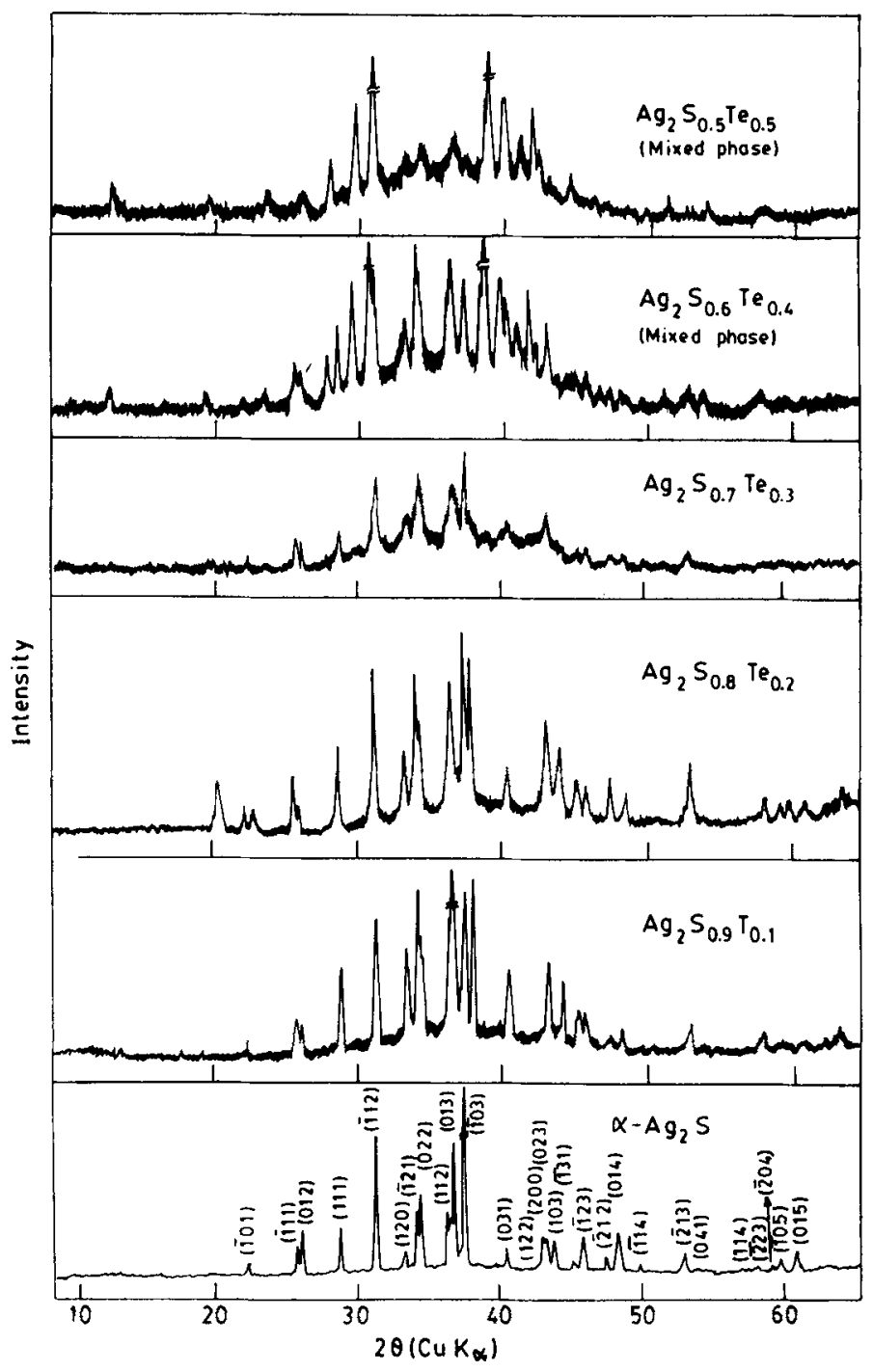

Figure 1. $X$-ray powder diffraction patterns of unannealed $\alpha-A_{g_{2}} S$ and solid solutions $\mathrm{Ag}_{2} \mathrm{~S}_{1-x} \mathrm{Te}_{x}(x \leqslant 0 \cdot 5)$.

Furthermore, all these phase transitions are reversible but are not as sharp as the parent compounds. It is interesting to note that the solid solution with $x=0.4$ shows only one phase transition, while the composition $x=0.5$ gives almost negligible enthalpy of phase transformation though mixtures of two phases are seen to be present in both the cases in their powder X-ray diffractograms.

Figure 4 shows the high temperature ( $523 \mathrm{~K}$ ) PXD patterns of $\mathrm{Ag}_{2} \mathrm{~S}$ and $\mathrm{Ag}_{2} \mathrm{Te}$ along with the four representative solid solutions. Of these the $\mathrm{Ag}_{2} \mathrm{~S}$ and $\mathrm{Ag}_{2} \mathrm{Te}$ diffractograms resemble the bcc and fcc phases as reported in the powder diffraction files of inorganic compounds (4-0774 and 6-0575), while all the solid solutions but for $x=0.2$ show an amorphous diffraction pattern. However, the solid solutions on cooling show poor crystallinity. 
Table 1. Indexed X-ray lines of $\mathrm{Ag}_{2} \mathrm{~S}$ and $\mathrm{Ag}_{2} \mathrm{~S}_{0.8} \mathrm{Te}_{0.2}$ (refined by least square method).

\begin{tabular}{|c|c|c|c|c|c|c|}
\hline \multicolumn{4}{|c|}{$\mathrm{Ag}_{2} \mathrm{~S}$} & \multicolumn{3}{|c|}{$\mathrm{Ag}_{2} \mathrm{~S}_{0.8} \mathrm{Te}_{0.2}$} \\
\hline$h k l$ & $d_{\text {obs }}$ & $d_{\mathrm{cal}}$ & $I_{\mathrm{obs}}$ & $d_{\mathrm{obs}}$ & $d_{\text {cal }}$ & $I_{\mathrm{obs}}$ \\
\hline & & & & $4 \cdot 291$ & - & 26 \\
\hline \multirow[t]{2}{*}{101} & 3.952 & 3.953 & 4 & 3.952 & 3.960 & 16 \\
\hline & & & & 3.850 & & 14 \\
\hline$\overline{1} 11$ & 3.440 & 3.434 & 10 & 3.434 & & 32 \\
\hline 012 & $3 \cdot 389$ & $3 \cdot 388$ & 17 & 3.376 & 3.378 & 14 \\
\hline 111 & 3.079 & 3.080 & 17 & 3.079 & 3.078 & 46 \\
\hline$\overline{1} 12$ & $2 \cdot 840$ & 2.837 & 53 & 2.886 & 2.837 & 88 \\
\hline- & 2.683 & $2 \cdot 644$ & 2 & & & \\
\hline 120 & 2.667 & 2.664 & 6 & 2.663 & 2.664 & 35 \\
\hline- & 2.644 & - & 2 & & & \\
\hline$\overline{1} 21$ & $2 \cdot 607$ & $2 \cdot 606$ & 22 & 2.607 & 2.607 & 79 \\
\hline 022 & $2 \cdot 582$ & 2.586 & 29 & 2.585 & 2.581 & 50 \\
\hline 121 & & & & 2.442 & 2.440 & 69 \\
\hline 112 & 2.458 & - & 22 & & & \\
\hline 013 & 2.426 & 2.425 & 48 & $2 \cdot 426$ & 2.417 & 48 \\
\hline$\overline{103}$ & $2 \cdot 383$ & $2 \cdot 384$ & 100 & $2 \cdot 385$ & $2 \cdot 380$ & 100 \\
\hline- & $2 \cdot 257$ & - & 3 & & & \\
\hline 031 & $2 \cdot 214$ & $2 \cdot 215$ & 8 & $2 \cdot 214$ & $2 \cdot 214$ & 23 \\
\hline 122 & 2.094 & 2.095 & 4 & & & \\
\hline 200 & 2.085 & 2.081 & 13 & 2.085 & 2.085 & 51 \\
\hline 023 & 2.076 & 2.074 & 12 & & & \\
\hline 103 & 2.051 & 2.051 & 10 & 2.045 & 2.045 & 37 \\
\hline$\overline{13} 1$ & 1.991 & 1.995 & 4 & 1.994 & 1.995 & 16 \\
\hline$\overline{1} 23$ & 1.965 & 1.964 & 12 & 1.967 & 1.962 & 16 \\
\hline 212 & 1.903 & 1.901 & 6 & 1.903 & 1.903 & 24 \\
\hline 014 & 1.870 & 1.870 & 14 & 1.868 & 1.863 & 14 \\
\hline$\overline{1} 14$ & 1.817 & 1.818 & 3 & & & \\
\hline$\overline{2} 13$ & 1.719 & 1.717 & 8 & 1.717 & $1 \cdot 718$ & 33 \\
\hline 041 & 1.692 & 1.692 & 2 & & & \\
\hline 114 & 1.610 & 1.612 & 1 & & & \\
\hline$\overline{1} 41$ & 1.585 & 1.587 & 1 & & & \\
\hline$\overline{2} 23$ & 1.579 & 1.578 & 3 & 1.580 & 1.579 & 14 \\
\hline$\overline{2} 04$ & 1.552 & 1.550 & 2 & 1.555 & 1.555 & 12 \\
\hline$\overline{1} 05$ & 1.542 & 1.542 & 6 & 1.541 & 1.538 & 10 \\
\hline 015 & 1.517 & 1.516 & 8 & 1.514 & 1.510 & 14 \\
\hline$\overline{1} 34$ & & & & 1.459 & 1.458 & 13 \\
\hline & & & & - & - & 22 \\
\hline
\end{tabular}

The PXD patterns and the phase transition temperatures of the annealed samples of the parent compounds and their solid solutions of compositions $x=0.1$ and 0.8 are the same as of the unannealed sample. For the intermediate compositions $(x=0.4-0.6)$, diffused patterns were observed similar to the one reported by Koji and Iida (1985) for the samples prepared by conventional solid-state reactions. For the compositions $x=0.2,0.3$ and 0.7 , the PXD patterns are poorly crystalline with a broad phase transition as shown in figure 5 . 


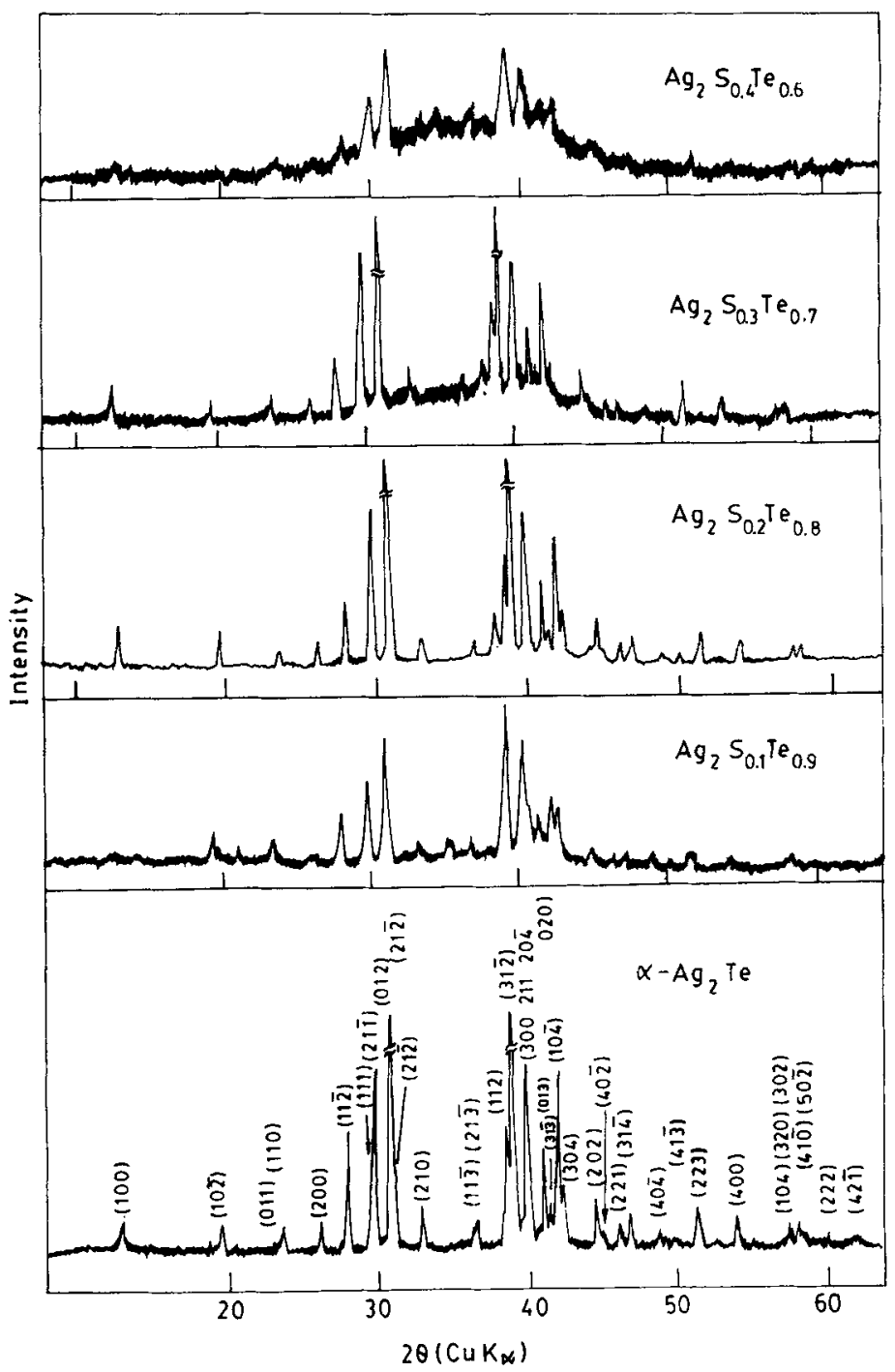

Figure 2. X-ray powder diffraction patterns of unannealed $\alpha-\mathrm{Ag}_{2} \mathrm{Te}$ and solid solutions $\mathrm{Ag}_{2} \mathrm{~S}_{1-x} \mathrm{Te}_{x}(x \geqslant 0 \cdot 6)$.

Table 2. Indexed $\mathrm{X}$-ray lines of $\mathrm{Ag}_{2} \mathrm{Te}$ and $\mathrm{Ag}_{2} \mathrm{~S}_{0 \cdot 2} \mathrm{Te}_{0 \cdot 8}$.

\begin{tabular}{cccccccc}
\hline & \multicolumn{3}{c}{$\mathrm{Ag}_{2} \mathrm{Te}$} & & & \multicolumn{3}{c}{$\mathrm{Ag}_{2} \mathrm{~S}_{0.2} \mathrm{Te}_{0.8}$} \\
\hline$h k l$ & $d_{\mathrm{obs}}$ & $d_{\text {cal }}$ & $I_{\text {obs }}$ & & $d_{\text {obs }}$ & $d_{\text {cal }}$ & $I_{\text {obs }}$ \\
\hline 100 & 6.810 & 6.779 & 11 & 6.733 & 6.760 & 13 \\
$10 \overline{2}$ & 4.495 & 4.489 & 8 & 4.484 & 4.484 & 10 \\
$11 \overline{1}$ & 3.834 & 3.840 & 3 & & 3.836 & - \\
002 & - & - & - & 3.754 & 3.758 & 5 \\
110 & 3.739 & 3.742 & 7 & 3.723 & 3.734 & 6 \\
\hline
\end{tabular}


Table 2. (Continued)

\begin{tabular}{|c|c|c|c|c|c|c|}
\hline \multicolumn{4}{|c|}{$\mathrm{Ag}_{2} \mathrm{Te}$} & \multicolumn{3}{|c|}{$\mathrm{Ag}_{2} \mathrm{~S}_{0.2} \mathrm{Te}_{0.8}$} \\
\hline$h k l$ & $d_{\mathrm{ots}}$ & $d_{\text {cal }}$ & $I_{\mathrm{obs}}$ & $d_{\mathrm{obs}}$ & $d_{\text {cal }}$ & $I_{\mathrm{obs}}$ \\
\hline 200 & 3.389 & $3 \cdot 389$ & 10 & $3 \cdot 700$ & 3.380 & 9 \\
\hline $11 \overline{2}$ & 3.175 & $3 \cdot 174$ & 29 & $3 \cdot 159$ & $3 \cdot 168$ & 24 \\
\hline 111 & 3.013 & 3.007 & 40 & - & 3.000 & - \\
\hline $21 \overline{1}$ & 2.993 & 2.995 & 62 & 2.979 & 2.986 & 57 \\
\hline 012 & $2 \cdot 880$ & $2 \cdot 881$ & 100 & - & $2 \cdot 879$ & - \\
\hline $21 \overline{2}$ & $2 \cdot 858$ & $2 \cdot 870$ & 30 & 2.867 & $2 \cdot 858$ & 100 \\
\hline 210 & 2.698 & $2 \cdot 706$ & 14 & 2.691 & $2 \cdot 698$ & 9 \\
\hline $11 \overline{3}$ & $2 \cdot 452$ & 2.452 & 9 & 2.447 & 2.449 & 6 \\
\hline $21 \overline{3}$ & 2.445 & - & - & - & $2 \cdot 440$ & \\
\hline- & - & - & - & 2.359 & - & 16 \\
\hline 112 & $2 \cdot 324$ & $2 \cdot 324$ & 44 & $2 \cdot 315$ & $2 \cdot 322$ & 35 \\
\hline $31 \overline{2}$ & $2 \cdot 308$ & $2 \cdot 309$ & 100 & $2 \cdot 295$ & $2 \cdot 301$ & 85 \\
\hline 300 & - & $2 \cdot 261$ & - & - & $2 \cdot 253$ & \\
\hline 211 & 2.254 & $2 \cdot 251$ & 64 & - & $2: 248$ & \\
\hline $20 \overline{4}$ & & 2.245 & - & $2 \cdot 242$ & $2 \cdot 240$ & 54 \\
\hline 020 & - & $2 \cdot 240$ & - & - & $2 \cdot 240$ & \\
\hline 013 & $2 \cdot 189$ & $2 \cdot 188$ & 36 & $2 \cdot 186$ & $2 \cdot 187$ & 28 \\
\hline $31 \overline{3}$ & $2 \cdot 171$ & $2 \cdot 174$ & 16 & $2 \cdot 166$ & $2 \cdot 167$ & 8 \\
\hline 021 & - & $2 \cdot 147$ & - & - & $2 \cdot 147$ & - \\
\hline 121 & $2 \cdot 145$ & $2 \cdot 145$ & 60 & - & $2 \cdot 145$ & \\
\hline $10 \overline{4}$ & - & $2 \cdot 136$ & - & $2 \cdot 137$ & $2 \cdot 135$ & 45 \\
\hline \multirow[t]{2}{*}{$30 \overline{4}$} & $2 \cdot 125$ & $2 \cdot 125$ & 23 & $2 \cdot 117$ & $2 \cdot 118$ & 16 \\
\hline & - & - & - & 2.041 & - & 4 \\
\hline 202 & 2.025 & 2.026 & 16 & 2.026 & 2.024 & 16 \\
\hline $40 \overline{2}$ & $2 \cdot 010$ & 2.007 & 3 & $2 \cdot 006$ & $2 \cdot 003$ & 4 \\
\hline $22 \overline{1}$ & 1.961 & 1.960 & 6 & 1.957 & 1.957 & 7 \\
\hline $11 \overline{4}$ & 1.935 & 1.929 & 10 & - & 1.927 & \\
\hline $31 \overline{4}$ & - & 1.920 & - & 1.927 & 1.915 & 10 \\
\hline $40 \overline{4}$ & 1.864 & 1.864 & 5 & 1.855 & 1.856 & 4 \\
\hline- & - & - & - & 1.852 & - & 1 \\
\hline 113 & 1.850 & 1.841 & 3 & 1.841 & 1.840 & 1 \\
\hline $41 \overline{3}$ & 1.826 & 1.827 & 4 & 1.817 & 1.820 & 3 \\
\hline $12 \overline{3}$ & 1.779 & 1.779 & 14 & - & $1 \cdot 778$ & - \\
\hline $22 \overline{3}$ & - & 1.777 & - & 1.771 & 1.775 & 11 \\
\hline 014 & 1.738 & 1.733 & 3 & 1.734 & $1 \cdot 173$ & 8 \\
\hline- & - & - & - & 1.728 & - & 1 \\
\hline $32 \overline{1}$ & - & 1.694 & & 1.692 & 1.691 & 8 \\
\hline 400 & 1.695 & 1.695 & 10 & - & 1.690 & \\
\hline 104 & 1.604 & 1.600 & 5 & 1.600 & 1.600 & 4 \\
\hline 410 & 1.587 & 1.586 & 8 & 1.585 & 1.582 & 4 \\
\hline $50 \overline{2}$ & - & 1.581 & - & 1.575 & 1.575 & 1 \\
\hline $12 \overline{4}$ & - & 1.546 & - & - & 1.545 & - \\
\hline 324 & - & $1 \cdot 542$ & - & 1.538 & 1.540 & 1 \\
\hline 114 & 1.512 & 1.507 & 2 & 1.506 & 1.507 & 1 \\
\hline 222 & 1.502 & $1 \cdot 503$ & 3 & 1.499 & 1.502 & 1 \\
\hline
\end{tabular}


Table 2. (Continued)

\begin{tabular}{|c|c|c|c|c|c|c|}
\hline \multicolumn{4}{|c|}{$\mathrm{Ag}_{2} \mathrm{Te}$} & \multicolumn{3}{|c|}{$\mathrm{Ag}_{2} \mathrm{~S}_{0.2} \mathrm{Te}_{0.8}$} \\
\hline$h k l$ & $d_{\mathrm{obs}}$ & $d_{\mathrm{cal}}$ & $I_{\text {obs }}$ & $d_{\text {obs }}$ & $d_{\mathrm{cal}}$ & $I_{\text {obs }}$ \\
\hline $42 \overline{2}$ & - & 1.497 & - & - & 1.493 & \\
\hline $30 \overline{6}$ & - & 1.497 & - & - & 1.493 & \\
\hline $51 \overline{4}$ & - & 1.495 & - & - & 1.488 & \\
\hline 321 & 1.448 & 1.448 & 15 & 1.443 & 1.446 & 13 \\
\hline $42 \overline{1}$ & - & 1.445 & - & - & 1.441 & \\
\hline- & - & - & - & 1.408 & - & 1 \\
\hline $22 \overline{5}$ & - & 1.395 & - & 1.397 & $1-394$ & 12 \\
\hline 032 & - & $1 \cdot 388$ & - & $1 \cdot 391$ & $1 \cdot 389$ & 10 \\
\hline
\end{tabular}

Table 3. Lattice parameters of $\mathrm{Ag}_{2} \mathrm{~S}$ and solid solutions $\mathrm{Ag}_{2} \mathrm{~S}_{(1-x)} \mathrm{Te}_{x}(x \leqslant 0 \cdot 3)$.

\begin{tabular}{lcccccc}
\hline Compound & $\begin{array}{c}a \\
(\AA)\end{array}$ & $\begin{array}{c}b \\
(\AA)\end{array}$ & $\begin{array}{c}c \\
(\AA)\end{array}$ & $\beta$ & $\begin{array}{c}\text { Cell vol } \\
\left(\AA^{3}\right)\end{array}$ & $\begin{array}{c}\text { Density } \\
\left(\mathrm{d} / \mathrm{gcm}^{-3}\right)\end{array}$ \\
\hline $\mathrm{Ag}_{2} \mathrm{~S}$ & $4.222(9)$ & $6.93(2)$ & $7.88(0)$ & $99.58(8)$ & 227.34 & 7.239 \\
$\mathrm{Ag}_{2} \mathrm{~S}_{0.9} \mathrm{Te}_{0.1}$ & $4.23(3)$ & $6.93(6)$ & $7.92(7)$ & $100 \cdot 1(4)$ & 228.57 & 7.478 \\
$\mathrm{Ag}_{2} \mathrm{~S}_{0.8} \mathrm{Te}_{0.2}$ & $4.23(2)$ & $6.93(4)$ & $7.85(4)$ & $99.7(1)$ & 226.82 & 7.815 \\
$\mathrm{Ag}_{2} \mathrm{~S}_{0.7} \mathrm{Te}_{0.3}$ & $4.23(3)$ & $6.94(4)$ & $7.86(5)$ & $99.9(2)$ & 227.37 & 8.075
\end{tabular}

Table 4. Lattice parameters of $\mathrm{Ag}_{2} \mathrm{Te}$ and solid solutions $\mathrm{Ag}_{2} \mathrm{~S}_{(1-x)} \mathrm{Te}_{x}(x \geqslant 0.6)$.

\begin{tabular}{lcccccc}
\hline Compound & $\begin{array}{c}a \\
(\AA)\end{array}$ & $\begin{array}{c}b \\
(\AA)\end{array}$ & $\begin{array}{c}c \\
(\AA)\end{array}$ & $\beta$ & $\begin{array}{c}\text { Cell vol } \\
\left(\AA^{3}\right)\end{array}$ & $\begin{array}{c}\text { Density } \\
\left(8 \mathrm{~cm}^{-3}\right)\end{array}$ \\
\hline $\mathrm{Ag}_{2} \mathrm{Te}$ & $8.10(2)$ & $4.488(9)$ & $8.98(2)$ & $123.15(7)$ & 273.32 & 8.343 \\
$\mathrm{Ag}_{2} \mathrm{Te}_{0.9} \mathrm{~S}_{0.1}$ & $0.07(3)$ & $4.47(2)$ & $8.94(3)$ & $123.0(7)$ & 270.46 & 8.196 \\
$\mathrm{Ag}_{2} \mathrm{Te}_{0.8} \mathrm{~S}_{0.2}$ & $8.10(2)$ & $4.488(9)$ & $8.98(2)$ & $123.0(1)$ & 273.78 & 7.875 \\
$\mathrm{Ag}_{2} \mathrm{Te}_{0.7} \mathrm{~S}_{0.3}$ & $8.08(2)$ & $4.47(2)$ & $8.97(3)$ & $123.04(9)$ & 270.98 & 7.646 \\
$\mathrm{Ag}_{2} \mathrm{Te}_{0.6} \mathrm{~S}_{0.4}$ & $8.08(2)$ & $4.47(1)$ & $8.97(3)$ & $123.25(7)$ & 270.94 & 7.414 \\
\hline
\end{tabular}

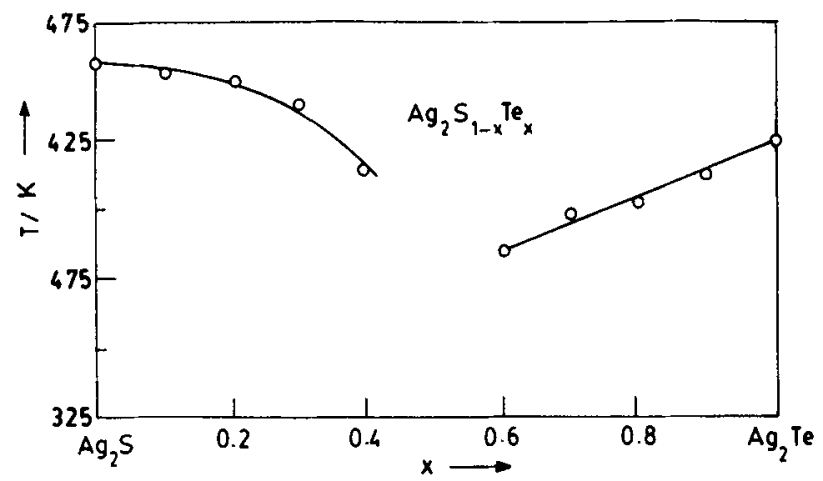

Figure 3. Phase transition temperature of $\mathrm{Ag}_{2} \mathrm{~S}$ and $\mathrm{Ag}_{2} \mathrm{Te}$ and their solid solutions $\mathrm{Ag}_{2} \mathrm{~S}_{1-x} \mathrm{Te}_{x}$ as a function of $x$. 


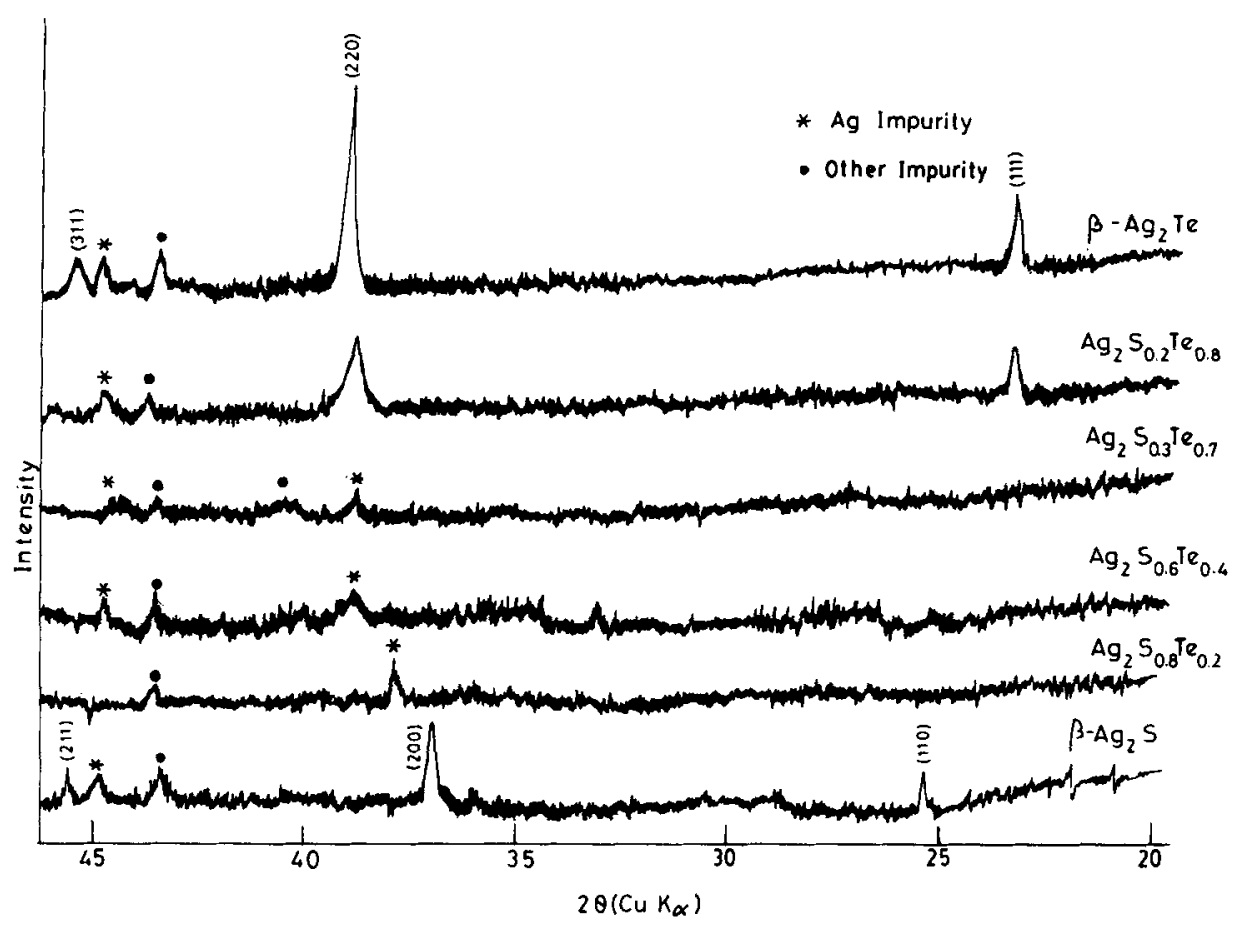

Figure 4. X-ray powder diffraction patterns of $\beta-\mathrm{Ag}_{2} \mathrm{~S}, \beta-\mathrm{Ag}_{2} \mathrm{Te}$ and $\mathrm{Ag}_{2} \mathrm{~S}_{1-x} \mathrm{Te}_{x}$ at $523 \mathrm{~K}$.

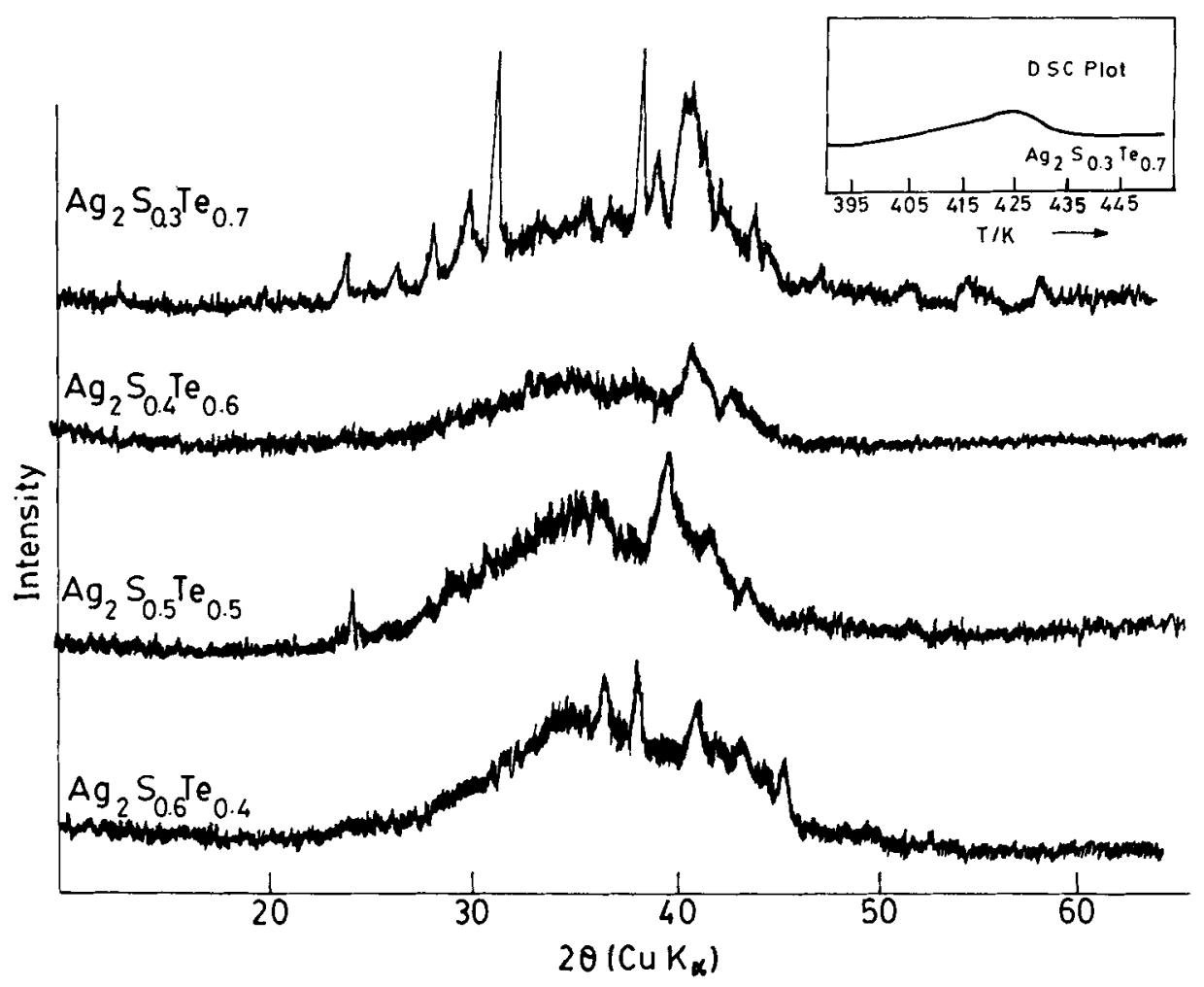

Figure 5. X-ray powder diffraction patterns of annealed $\mathrm{Ag}_{2} \mathrm{~S}_{1-x} \mathrm{Te}_{x}(x=0.4-0.7)$; the inset shows a typical DSC curve for the solid solution of $x=0.7$. 


\section{References}

Koji H and Iida K 1985 J. Phys. Soc. Jpn 542218

Kulifay M S 1962 J. Am. Chem. Soc. 834961

Miyatani S 1958 J. Phys. Soc. Jpn 13341

Miyatani S 1959 J. Phys. Soc. Jpn 141634

Miyatani S 1960 J. Phys. Soc. Jpn 151586

Pass G and Sutcliffe H 1968 Practical inorganic chemistry (London: Chapman and Hall)

Shukla A K and Schmalzried H 1979 Z. Phys. Chem. 59118

Shukla A K, Vasan H N and Rao C N R 1981 Proc. R. Soc. (London) A376 619

Sohege J and Funke K 1984 Ber. Bunsenges. Phys. Chem. 88657

Sohege J and Funke K 1989 Ber. Bunsenges. Phys. Chem. 93115

Wagner C 1953 J. Chem. Phys. 211819 